\title{
CANNULATION STRATEGY FOR AORTIC ARCH RECONSTRUCTION USING DEEP HYPOTHERMIC CIRCULATORY ARREST
}

\author{
Diane de Zéicourt, PhD ${ }^{1}$, Philsub Jung ${ }^{1}$, Marc Horner ${ }^{2}$, Kerem Pekkan, PhD ${ }^{3}$, Kirk R. \\ Kanter, $\mathbf{M D}^{4}$, and $\mathbf{A j i t} \mathbf{P}$. Yoganathan, $\mathbf{P h D}^{1}$ \\ ${ }^{1}$ Wallace H. Coulter Department of Biomedical Engineering, Georgia Institute of Technology, \\ Atlanta, GA \\ ${ }^{2}$ ANSYS Inc., Evanston, IL \\ ${ }^{3}$ College of Engineering at Carnegie Mellon University, Pittsburgh, PA \\ ${ }^{4}$ Division of Cardiothoracic Surgery, Emory University, Atlanta, GA
}

\section{Abstract}

\begin{abstract}
Background-Aortic arch reconstruction in neonates is commonly performed using deep hypothermic circulatory arrest. However, concerns have arisen regarding potential adverse neurologic outcomes from this complex procedure, raising questions as to best arterial cannulation approach for cerebral perfusion and effective systemic hypothermia. In this study, we use computational fluid dynamics to investigate the impact of different cannulation strategies in neonates.
\end{abstract}

\begin{abstract}
Methods-Using a realistic hypoplastic neonatal aorta template as the base geometry, four different cannulation options were investigated: 1) right innominate artery, 2) innominate root, 3) patent ductus arteriosus (PDA), or 4) both innominate root and PDA. Performance was evaluated based on the numerically predicted cerebral and systemic flow distributions compared with physiological perfusion under neonatal conditions.
\end{abstract}

Results-The four cannulation strategies were associated with different local hemodynamics, but this did not translate into any significant effect on the measured flow distributions. The largest difference only represented $0.8 \%$ of the cardiac output and was measured in the innominate artery, which received $23.2 \%$ of the cardiac output in Option 3 vs. 24\% in Option 4. PA snaring benefited all systemic vessels uniformly.

Conclusion-Due to the very high vascular resistances in neonates, flow distribution to the different vascular beds was dictated by the downstream vascular resistances rather than the cannulation strategy, allowing the surgical team to choose their method of preference. However, patients with aortic coarctation warrant further investigation and will most likely benefit from a two cannulae approach (Option 4).

(C) 2012 The Society of Thoracic Surgeons. Published by Elsevier Inc. All rights reserved.

Corresponding Author: Ajit P. Yoganathan, Ph.D., Wallace H. Coulter School of Biomedical Engineering, Georgia Institute of Technology and Emory University, 313 Ferst Drive Atlanta, GA 30332-0535, TEL: 404-894-2849, FAX: 404-894-4243, ajit.yoganathan@bme.gatech.edu.

Publisher's Disclaimer: This is a PDF file of an unedited manuscript that has been accepted for publication. As a service to our customers we are providing this early version of the manuscript. The manuscript will undergo copyediting, typesetting, and review of the resulting proof before it is published in its final citable form. Please note that during the production process errors may be discovered which could affect the content, and all legal disclaimers that apply to the journal pertain. 


\section{Keywords}

Aortic operation; Computer applications; CHD, hypoplastic left heart syndrome; Hypothermia/ circulatory arrest; Neonate

\section{Introduction}

The use of deep hypothermic cardiac arrest (DHCA) has been established as a standard procedure for neonates born with a hypoplastic left heart syndrome who require surgical repair of a hypoplastic aortic arch. Unfortunately, DHCA has also been associated with significant complications including impaired neurodevelopment [1], and non-neurologic morbidities such as pulmonary and renal dysfunction [2], and multi-organ failure. While the complications associated with DHCA are no doubt multifactorial, proper perfusion of all vascular beds during the cooling process is a primary requirement, ensuring that not only the brain but also all peripheral organs are sufficiently hypothermic.

Hypoplastic aortic arches represent nonstandard and complex surgical anatomies that lend themselves to several possibilities for arterial cannulation, including insertion into the: a) innominate artery [3]; b) right subclavian artery [4]; or c) patent ductus arteriosus (PDA) [5]. The pulmonary arteries (PAs) or the PDA are also commonly snared in an effort to further increase systemic blood flow. However, the exact effect of the different options on the actual cerebral perfusion in HLHS patients remains unknown. Likewise, whether or not PA or PDA snaring truly brings a benefit in cerebral perfusion remains to be demonstrated.

Experimental and computational bioengineering studies have begun to address these issues, investigating the impact of cannula design [6] and location [7] on perfusion characteristics in normal aortic arches. The recent study of Kauffman et al. [7], for example, compares the hemodynamic characteristics of a standard bypass configuration with different cannula insertion points and angles for a healthy aortic arch configuration. Yet, the geometry of hypoplastic aortic arches and associated hemodynamics [8] drastically differ from that of normal aortic arch configuration and is highly variable from patient-to-patient. In addition, the neonatal circulation is characterized by a significantly lower cardiac output and very high vascular resistances compared to adults. These challenges limit the generalization of the former studies performed on normal adult aortas to patients with congenital hypoplastic left heart syndrome.

The lack of bioengineering studies on neonatal arches may in part be explained by the difficulty to access in vivo anatomies and precise flow information. Indeed, exposure to radiation for computed tomography is not desirable at such a young age, and sedation is required for MRI. Likewise the transcranial and intraoperative pulsed Doppler ultrasound measurements lead to large measurement errors due to the unclear probe orientation on tiny great vessels. However, to achieve a comparative assessment on the impact of cannulation strategies on hemodynamics, the inclusion of all patient-specific details may not yet be necessary and significant insights may still be gained through a realistic idealized hypoplastic aortic arch template. Therefore, in this manuscript, an idealized computational test-bed is developed to isolate the effect of different cannulation locations and PA snaring on the flow distribution of a neonatal hypoplastic aortic arch during systemic cooling. This study builds upon our previous work [8,9], which is the only bioengineering study available in literature that investigated the hemodynamics of hypoplastic and congenitally defective aortas. 


\section{Material and Methods}

\section{Cannula Insertion Locations}

The baseline aortic arch anatomy, inclusive of the patent ductus arteriosus (PDA), corresponds to the realistically idealized hypoplastic aortic arch template reconstructed by Pekkan et al. [8] (Figure 1, top panel), based on the vessel dimensions reported by three pediatric cardiologists and extensive literature search (for example Ilbawi et al. [10]). Four common cannula insertion locations (with cannula tips of $4 \mathrm{~mm}$ in diameter) were incorporated into this geometry (Figure 1). Option 1 illustrates a cannula inserted into the innominate artery. Because cannula insertions into or around the innominate artery may be expected to strongly favor flow to that vessel, the cannula in Option 1 was inserted into the proximal section of the vessel to determine the lower bound of the expected innominate flow rate. Option 2 presents a variation of that design with the cannula inserted into the root of the innominate artery and oriented towards the branching between the aortic arch and the left carotid artery. In Option 3, the cannula inserted through the PDA. Finally, Option 4 models a bifurcated cannula with two tips; one inserted into the innominate root and one through the PDA, thereby combining the second and third configurations.

\section{CFD Simulations}

The computational method used in this study has been validated against in vitro experiments [11] and in vivo piglet pressure data during bypass [8]. Briefly, all geometries were meshed in GAMBIT (ANSYS Inc., Canonsburg, PA) using the HexCore/TGrid scheme. A nonuniform mesh resolution was used to properly capture the regions of high velocity while minimizing the computational cost. A preliminary mesh refinement investigation indicated that the pressure and flow distributions were grid-insensitive when using a grid spacing of $0.15 \mathrm{~mm}$ or lower in the high velocity regions. Accordingly, a spacing of $0.15 \mathrm{~mm}$ was applied to the regions of high velocity, while a spacing of $0.3 \mathrm{~mm}$ or $0.6 \mathrm{~mm}$ was used in the regions of lower velocity such as pulmonary arteries or ascending aorta, resulting in approximately 1 million elements for each configuration. The flow simulations were conducted in FLUENT (ANSYS Inc., Canonsburg, PA), using an unsteady, $2^{\text {nd }}$ order, implicit formulation $[8,11]$. The convergence criterion was set to $10 \mathrm{E}^{-5}$ for all degrees-offreedom.

The simulation set-up and boundary conditions are summarized in Figure 2. Since cardiopulmonary bypass delivers a non-pulsatile flow rate, neglecting the aortic wall motion is considered a fair assumption and the vessel walls were modeled as rigid no-slip boundaries. Resistance boundary conditions were imposed at the outlets to represent the downstream systemic vasculature. The resistance values were calculated by conducting a set of preliminary simulations without any cannula until the computed mass flow rate at each outlet matched physiological flow distributions for neonates. In these preliminary simulations, blood flow rate was set to $0.87 \mathrm{~L} / \mathrm{min}$, representing the cardiac output for a neonate infant of $3.5 \mathrm{~kg}$ [12]. The target physiological neonatal flow distributions were determined assuming an equal distribution to the superior and inferior systemic beds, and that before closure of the PDA about $30 \%$ of the flow went to the pulmonary arteries. Retrograde perfusion of the coronary arteries (4 to $5 \%$ of the cardiac output) was modeled by setting an outflow resistance boundary condition at the hypoplastic ascending aorta crosssection. Matching the desired mass flow splits constrains the pressure gradients across the aorta but leaves one degree of freedom for the absolute pressure values. The resistances were thus adjusted for a mean aortic pressure of $50 \mathrm{mmHg}$.

The vascular resistances defined in the preliminary simulations were then assumed to hold constant during perfusion. The perfusion flow rate was set to $0.525 \mathrm{~L} / \mathrm{min}$ as is customary 
during DHCA. For Option 4, with a dual cannula, the inflow rate was divided equally between the two cannulae. PA snaring was modeled by blocking off the pulmonary outlets (modeled as wall boundary conditions), while maintaining the systemic resistances constant. PDA snaring was not explicitly modeled as it was expected to yield flow distributions similar to those with PA snaring since both methods exclude flow from the PAs.

\section{Results \\ Vascular Resistances}

The neonatal resistances are 7000, 2370, 5923, 3933, 1189 and 2740 MPa.s.m ${ }^{-3}$ for the coronaries, innominate artery, left carotid, left subclavian, descending aorta and pulmonary arteries, respectively. This corresponds to global superior and inferior systemic vascular resistances of 148 and 149 Woods units, respectively. These values fall within close range to the ones reported by Lagana et al. [13] in their model of a neonatal Norwood circulation.

\section{Impact of Cannulation Location on Flow Distribution (PAs Snared)}

Simulation results for DHCA with the PAs snared are summarized in Table 2 in terms of blood flow distribution. Flow distributions measured in the neonatal hypoplastic aorta (without any cannula) with and without PA snaring are provided as reference. The corresponding flow pathways are illustrated in Figure 3 using 3D streamlines color-coded by velocity.

In all options, blood flow to the coronaries is ensured via retrograde perfusion of the hypoplastic ascending aorta (6.3\% to $6.6 \%$ of the flow). When the cannula was inserted into the innominate artery (Option 1), blood flow impinged against the vessel wall opposite to the insertion point and splits between the innominate and the other vessels (point S1). The innominate artery receives $24 \%$ of the blood, which is comparable to what was observed in the neonatal hypoplastic aorta with PA snared. The remaining $70 \%$ of the blood flowed into the aortic arch, creating a flow detachment and small recirculation region at the root of the left carotid artery. While this could have a detrimental effect for the perfusion of the left carotid, the jet momentum was here small enough for the flow to curve around the detachment region and perfuse the left carotid. Flow recirculation regions were also noted at the root of the left carotid and subclavian arteries, and along the inner wall of the aortic arch. Flow reattached in the descending aorta downstream of the PDA.

The flow structures observed when the cannula was approximately aligned with the axis of the ascending aorta (as in Option 2) were fairly comparable to those observed in Option 1, with the exception of the hemodynamics at the root of the innominate and left carotid artery. Blood split between the innominate and the outer wall of the aortic arch (point S1), and then impinged against the root of the left carotid (point S2) and subclavian arteries (point S3). These subsequent impingements allowed for a streamlined division of the blood flow between the different head and neck vessels.

The dynamics observed when inserting the cannula into the ductus (Option 3) were drastically different. Blood impingement onto the aortic wall led to a complex helical flow pattern throughout the arch, allowing for the perfusion of all vessels with few flow separation regions. In the dual cannulae approach (Option 4), blood coming through the ductus mostly perfused the descending aorta, while blood entering the innominate root was mostly distributed to the head-neck vessels and coronaries. The resulting flow structures combine the characteristics of Options 2 and 3. 
Despite significantly different flow structures, the most striking characteristic of these results is that the resulting flow distributions are all in close range of one another (Table 1), closely reproducing the flow distributions of the neonatal hypoplastic configuration.

\section{Effect of PA Snaring}

In order to quantify the impact of PA snaring on cerebral perfusion, flow distributions measured in Options 1-4 were compared to the pre-operative ones, i.e. without the PAs snaring (Table 1). Considering only the distribution of the systemic flow rate (i.e.

$\left.\%_{i}=\frac{Q_{i}}{C O-Q_{L P A}-Q_{R P A}}\right)$, we obtain the following distributions in the pre-operative configuration without snaring: $23.2 \%, 9.4 \%, 14.1 \%, 6.6 \%$ and $46.8 \%$ for the innominate, left carotid, left subclavian, coronaries and descending aorta respectively. These values are exactly the ones obtained when snaring the PAs in the control case, and very close to the ones measured in Options 1-4, implying that the blood flow excluded from the pulmonary circulation through snaring is uniformly distributed to all systemic vascular beds.

\section{Verification (4.5 L/min)}

As noted above, while the cannula insertion location had an impact on the local hemodynamics, it had little effect on the resultant flow distributions. This finding may appear to contradict previous literature, such as the study of Kaufmann et al. [7] which reports a clear impact of even small cannulation variations. However, these studies were conducted for adult applications, implying different perfusion flow rates and downstream vascular resistances. In order to verify our approach, an additional set of simulations was thus conducted, seeking to reproduce the flow conditions of [7]. These simulations do not carry any direct relevance to DHCA but are rather meant to verify that the different cannulation options would have yielded different perfusion patterns under adult conditions. As in [7], coronary blood flow was neglected, the inflow rate was $4.5 \mathrm{~L} / \mathrm{min}$. and the mean aortic pressure set to $60 \mathrm{mmHg}$. Target systemic flow distributions and corresponding outflow resistances are provided in Table 2 . These resistances correspond to an inferior and a global superior vascular resistance of 20 and 32 Woods unit, respectively.

The flow fields and flow distributions obtained with the four cannulation options, under adult flow conditions, are provided in Figure 4 and Table 2. The flow fields carry some resemblance to the ones observed in the DHCA simulations, but with increased flow disturbances and larger flow separation regions due to the higher jet momentum. More remarkably, there were measurable differences in flow distributions. In Option 1, the flow separation at the root of the left carotid obstructs the access to that vessel, which only receives $2.7 \%$ of the flow (vs. $8 \%$ in the reference case). Careful placement of the cannula as in Option 2 can help minimize such effect. Still both options 1 and 2 led to a significant increase of the innominate blood flow ( $34.5 \%$ or more) compared to the reference value of $20 \%$. In contrast, Options 3 and 4 yielded flow distributions in closer match to the control values. Option 4 again combined the characteristics of Options 2 and 3 inducing a slight increase in innominate flow rate $(24.2 \%$ vs. $20 \%)$ but still maintaining a proper perfusion of the lower systemic beds.

\section{Comment}

Although DHCA has played a central role in the development of surgical strategies to repair the aortic arch, it has concurrently been the subject of controversial discussions with an increasing emphasis placed on the post-operative neurological complications and significant non-neurologic morbidities. Multiple cannulation strategies have been discussed in literature $[3,4,5]$, seeking to ensure that all tissues are uniformly cooled thereby minimizing the 
consequences of ischemia-reperfusion. Snaring the PAs (or the PDA) is also commonly used to try to increase systemic perfusion during the cooling process. However, it is difficult to quantify the efficacy of these strategies in vivo, as it would require additional instrumentations in an already crowded and complex surgical space. As a result, neither the impact of the cannulation location nor that of PA snaring is properly understood. In this study, we compare three broad cannulation strategies, inserting a single cannula into the innominate artery (Options 1 and 2), or into the PDA (Option 3), or using two cannulae (Option 4); and quantify the benefits of PA snaring for each one of them.

Under neo-natal conditions, all options lead to fairly comparable flow distributions, despite different hemodynamics. This is due to two combined factors: a) the low momentum energy of the jet, due to the small perfusion flow rate $(0.525 \mathrm{~L} / \mathrm{min})$; and more importantly b) the high neonatal vascular resistances. Indeed, blood flow to a given vessel in the domain of interest experiences two serial resistances-the internal resistance of the connection (say the resistance encountered going from the cannula to the innominate outlet) and the resistance of the downstream vasculature. Because of the high neonatal vascular resistances imposed, the latter component dominates in all cases, leading to very low sensitivity to cannulation design. As an example, the internal resistance going from the cannula to the innominate was estimated to 229 and 317 MPa.s.m-3 in Options 1 and 3, respectively. The 88 MPa.s.m ${ }^{-3}$ difference was masked by the 2370 MPa.s. $\mathrm{m}^{-3}$ downstream vascular resistance. Conversely in the adult model of Kaufmann et al. [7] and our own validation case, the internal resistance is much higher (owing to higher flow disturbances because of the higher flow rates) and the downstream resistances are much lower, making the cannula placement and orientation a much more critical consideration.

These findings are of major clinical importance as it implies that in neonatal patients without further aortic arch complications, the surgeon should be able to use the cannulation strategy of his choice and yet achieve the flow distributions dictated by the downstream vascular resistances. In addition, comparison of the flow distributions with PA snaring to the control without snaring demonstrates that snaring the PAs benefits all systemic vascular beds equally, i.e. the amount of blood flow derived from the pulmonary circulation into a given vascular bed will be proportional to the resistance of that vascular bed. In particular, if some vascular beds become constricted faster than other ones, due to the effect of vasoconstrictors or to their cooling down, their resistance will increase and the distribution of the cold blood will be re-routed towards the vascular beds with a lower resistance (including those that have received vaso-dilators).

However, three things are important to keep in mind. First, the relative insensitivity to the cannulation approach only holds true under neonatal conditions, and not under adult conditions. Second, in all presented options, the cannula(e) only slightly protruded into the vessel and thereby did not obstruct flow towards any of the systemic vessels. However, if a cannula was inserted more deeply, then it could obstruct the flow and increase the local resistance, which could in turn influence the overall flow distributions. As an example, perfusion through a Goretex conduit sown to the innominate presents the advantage that it will not obstruct the innominate artery in any way, whereas a direct cannulation to that vessel should be performed without inserting the cannula too deeply. Similarly, cannulation into the ascending aorta carries the risk to block part of the retrograde perfusion of the coronaries if the hypoplastic ascending aorta is not sufficiently larger than the cannula. Along the same lines, the third point is that the aortic geometry presented herein featured an aorta that was hypoplastic but otherwise normal. In particular, it did not feature any aortic coarctation, which is present to some degrees in about half of the HLHS patients. For Options 1-3, any increase in resistance in the isthmus of the coarctation will bias flow towards the upper- or lower-body, depending on the point of insertion of the cannula. As 
illustrated in Figure 3, the two cannulae approach (Option 4) allows blood to be supplied both upstream and downstream of the coarctation, thereby minimizing the impact of the coarctation resistance and ensuring that the resultant flow distributions do not significantly deviate from their physiological values. While a dual cannulation approach is routinely used for patients with severe coarctation, having two independent blood sources may also provide an advantage for patients with mild coarctation as soon as there is a measurable arm-leg pressure difference, reflecting a significant increase in the isthmus resistance. Future work should incorporate varying degrees of aortic coarctation to assess the effectiveness of using two cannulae and quantify the impact of different cannula insertion depth into the ascending aorta and innominate artery.

\section{Limitations}

Idealized approaches are a perfect setting to conduct preliminary investigations, narrowing down the number of parameters to only those that seem to have the largest impact. Still the relevance of the associated findings should be carefully considered. The simplified neonatal aortic arch used herein was designed based on a careful review of the available literature and anatomical data. Furthermore, the different scenarios envisioned were meant to capture the effect of largely different strategies. The absence of differences between those scenarios implies that small changes in the local geometry are likely to have a minor, non-measurable impact on the measured performances. Beyond the geometrical simplifications, the downstream vasculatures are modeled by a fixed set of normalized resistances whereas, in vivo, these vascular resistances may vary with progressive hypothermia. As discussed above, this will have a direct impact on the flow distribution, redistributing the blood flow to the least constricted vessels. However, the variation of vascular tone during cardiopulmonary bypass and hypothermic cooling is not well understood. The next step would thus be to conduct concurrent clinical and computational investigations to enable more detailed modeling and correlate the two modalities.

\section{Conclusion}

This study sought to determine the cannulation strategy that will best distribute blood flow and to characterize the benefits of PA snaring during DHCA in HLHS patients. The relative resistance of the different vascular beds was found to be the strongest determinant for the flow distributions during cooling in neonates, due to the particularly high resistance of the neonatal vascular beds. As a consequence in patients without aortic coarctation or other vessel stenosis, the cannulation strategy had little impact on the global performance, allowing the surgeon to choose his method of preference (one or two cannulae, location and angle of insertion). Patients with an aortic coarctation will most likely present an exception to that rule and benefit from a two cannula approach (Option 4). Future work should thus incorporate varying degrees of aortic coarctation to assess the effectiveness of using two cannulae, and varying cannula insertion depth.

\section{Acknowledgments}

This study was supported by the NHLBI Grant HL67622.

\section{References}

1. Wypij D, Newburger JW, Rappaport LA, duPlessis AJ, Jonas RA, Wernovsky G, Lin M, Bellinger DC. The effect of duration of deep hypothermic circulatory arrest in infant heart surgery on late neurodevelopment: The Boston Circulatory Arrest Trial. Journal of Thoracic and Cardiovascular Surgery. 2003; 126(5):1397-1403. [PubMed: 14666011] 
2. Okita Y, Ando M, Minatoya K, Kitamura S, Takamoto S, Nakajima N. Predictive factors for mortality and cerebral complications in arteriosclerotic aneurysm of the aortic arch. Ann Thorac Surg. 1999; 67(1):72-78. [PubMed: 10086527]

3. Tchervenkov CI, Korkola SJ, Shum-Tim D. Surgical technique to avoid circulatory arrest and direct arch vessel cannulation during neonatal aortic arch reconstruction. Eur J Cardiothorac Surg. 2001; 19(5):708-710. [PubMed: 11343957]

4. Gerdes A, Joubert-Hubner E, Esders K, Sievers HH. Hydrodynamics of aortic arch vessels during perfusion through the right subclavian artery. Ann Thorac Surg. 2000; 69(5):1425-1430. [PubMed: 10881817]

5. Poirier NC, Drummond-Webb JJ, Hisamochi K, Imamura M, Harrison AM, Mee RB. Modified Norwood procedure with a high-flow cardiopulmonary bypass strategy results in low mortality without late arch obstruction. J Thorac Cardiovasc Surg. 2000; 120(5):875-884. [PubMed: 11044313]

6. Joubert-Huebner E, Gerdes A, Sievers HH. An in vitro evaluation of a new cannula tip design compared with two clinically established cannula-tip designs regarding aortic arch vessel perfusion characteristics. Perfusion. 2000; 15(1):69-76. [PubMed: 10676870]

7. Kaufmann TAS, Hormes M, Laumen M, Timms DL, Linde T, Schmitz-Rode T, Moritz A, Dzemali O, Steinseifer U. The Impact of Aortic/Subclavian Outflow Cannulation for Cardiopulmonary Bypass and Cardiac Support: A Computational Fluid Dynamics Study. Artificial Organs. 2009; 33(9):727-732. [PubMed: 19775264]

8. Pekkan K, Dur O, Sundareswaran K, Kanter K, Fogel M, Yoganathan A, Undar A. Neonatal aortic arch hemodynamics and perfusion during cardiopulmonary bypass. J Biomech Eng. 2008; 130(6): 061012. [PubMed: 19045541]

9. Pekkan K, Dasi LP, Nourparvar P, Yerneni S, Tobita K, Fogel MA, Keller B, Yoganathan A. In vitro hemodynamic investigation of the embryonic aortic arch at late gestation. J Biomech. 2008; 41(8):1697-1706. [PubMed: 18466908]

10. Ilbawi AM, Spicer DE, Bharati S, Cook A, Anderson RH. Morphologic study of the ascending aorta and aortic arch in hypoplastic left hearts: surgical implications. J Thorac Cardiovasc Surg. 2007; 134(1):99-105. [PubMed: 17599493]

11. Wang C, Pekkan K, de Zelicourt D, Horner M, Parihar A, Kulkarni A, Yoganathan AP. Progress in the CFD modeling of flow instabilities in anatomical total cavopulmonary connections. Ann Biomed Eng. 2007; 35(11):1840-1856. [PubMed: 17641974]

12. Walther FJ, Siassi B, Ramadan NA, Ananda AK, Wu PY. Pulsed Doppler determinations of cardiac output in neonates: normal standards for clinical use. Pediatrics. 1985; 76(5):829-833. [PubMed: 2932675]

13. Lagana K, Balossino R, Migliavacca F, Pennati G, Bove EL, de Leval MR, Dubini G. Multiscale modeling of the cardiovascular system: application to the study of pulmonary and coronary perfusions in the univentricular circulation. J Biomech. 2005; 38(5):1129-1141. [PubMed: 15797594] 


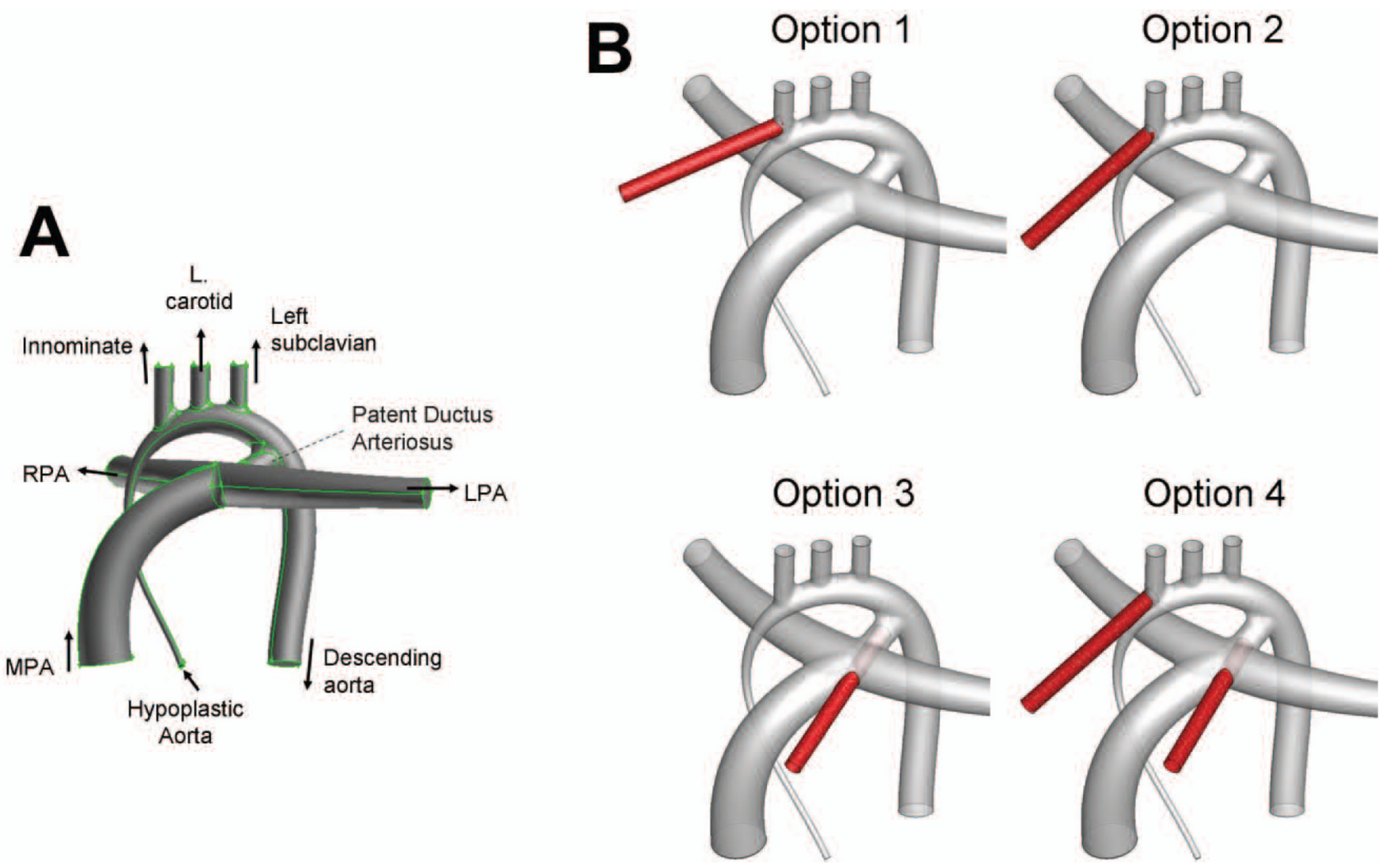

Figure 1.

A: Geometry of the idealized hypoplastic aorta. B: The four cannula insertion locations. The cannula is shown in red, while the hypoplastic aorta is shown in gray. 


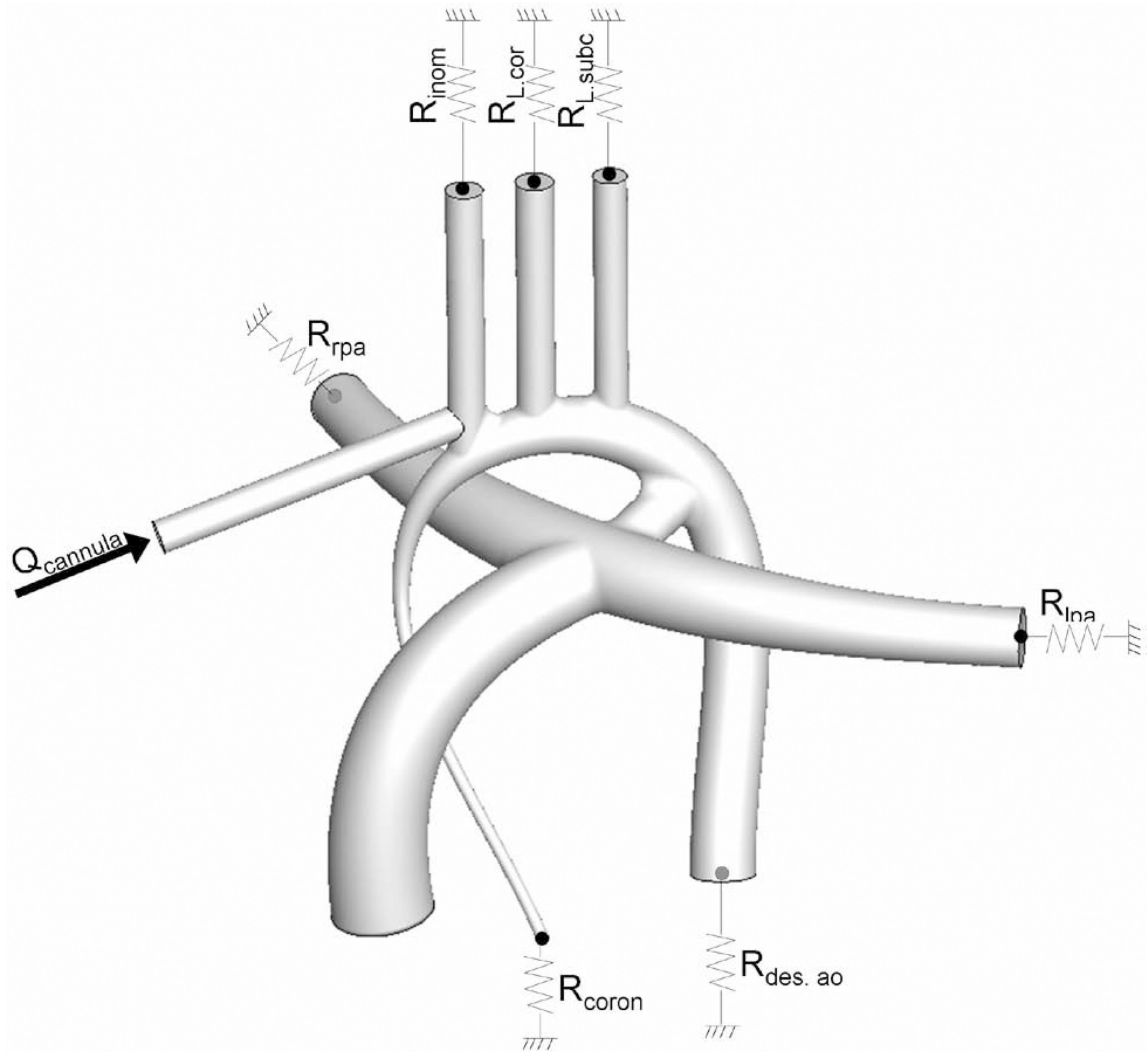

Figure 2.

Schematic representation of the inflow and outflow boundary conditions. 

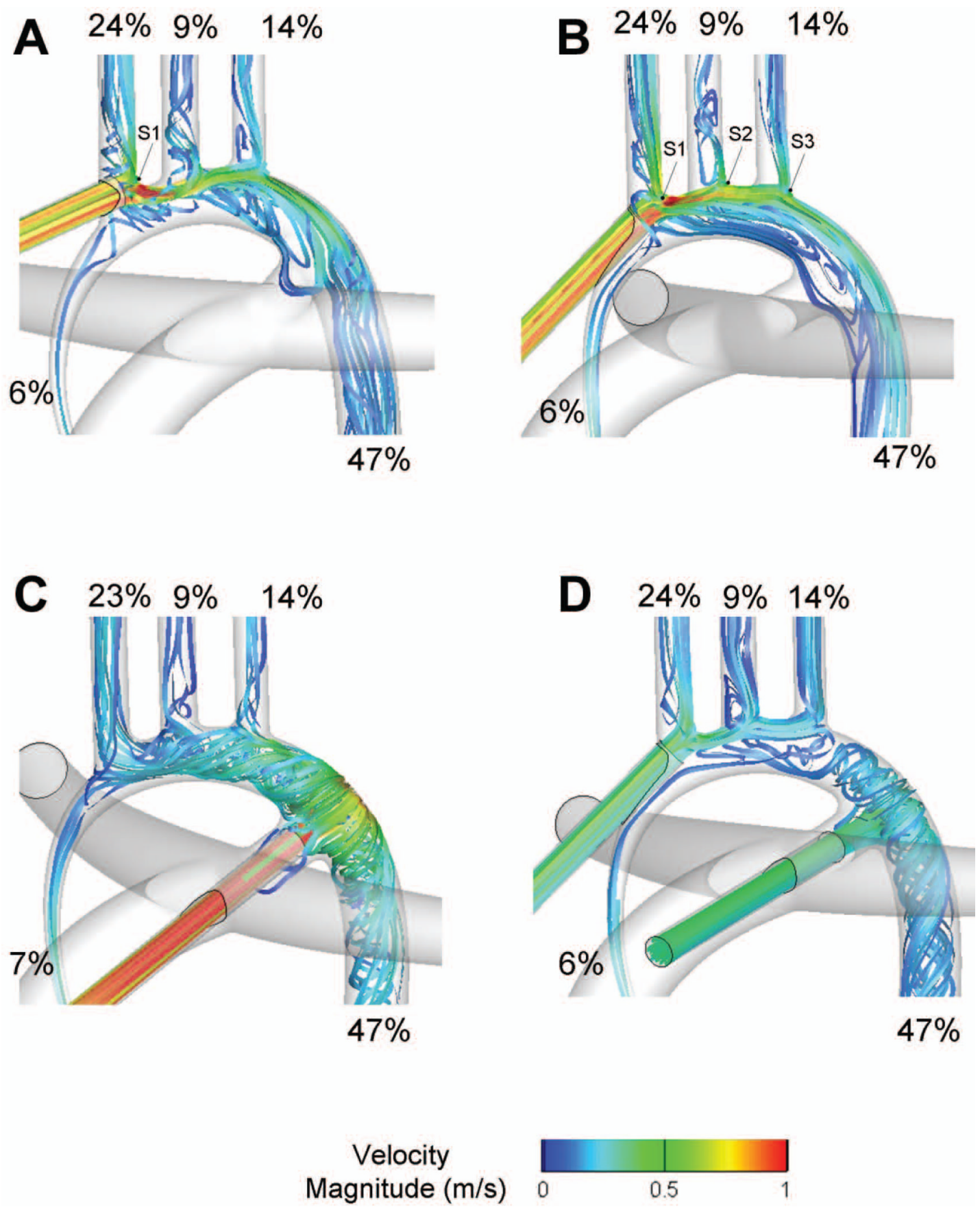

Figure 3.

Comparison of the flow distributions and velocity fields associated with the four cannulation options with PA snaring. S1, S2, S3: flow splitting points. A: Option 1; B: Option 2; C: Option 3; D: Option 4. 

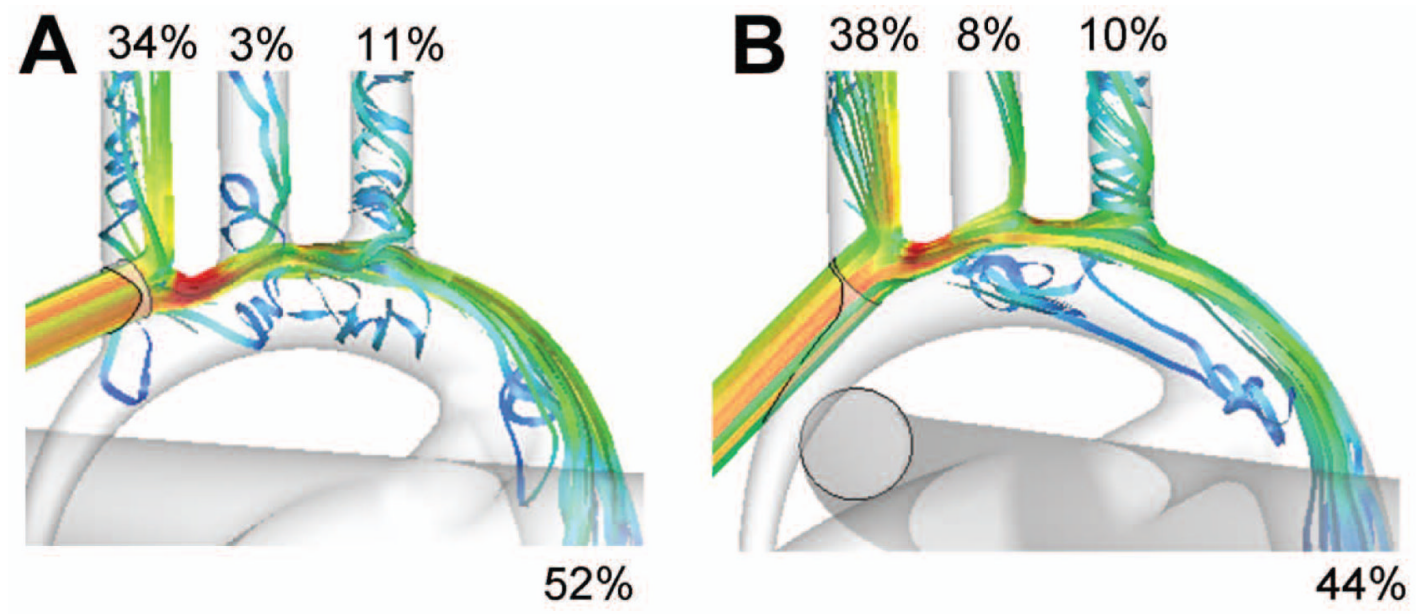
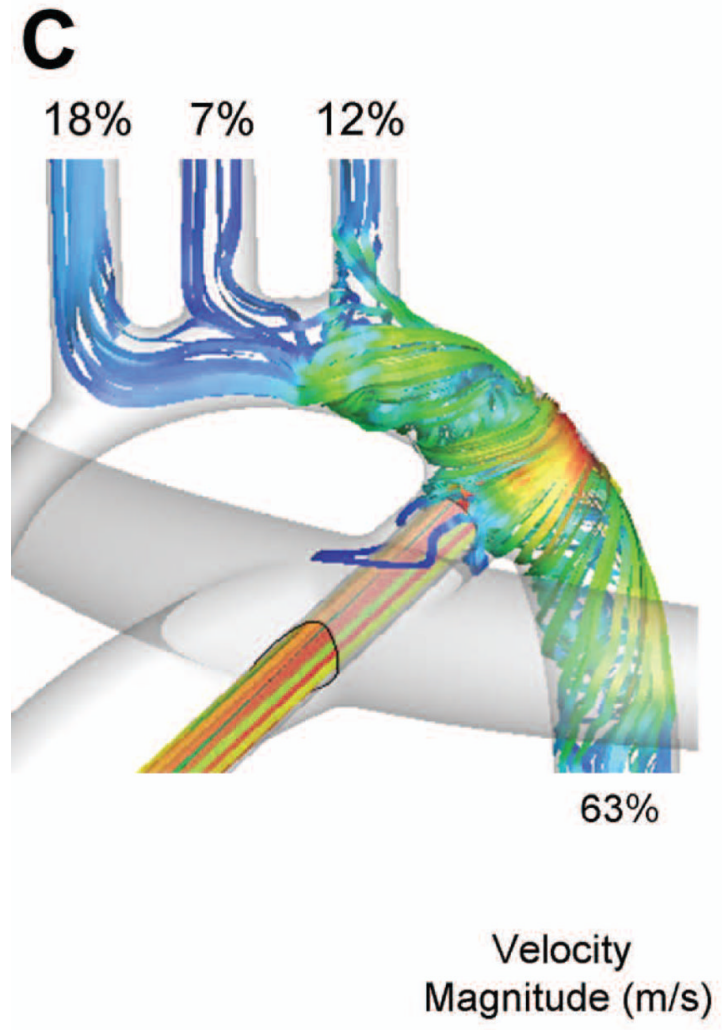
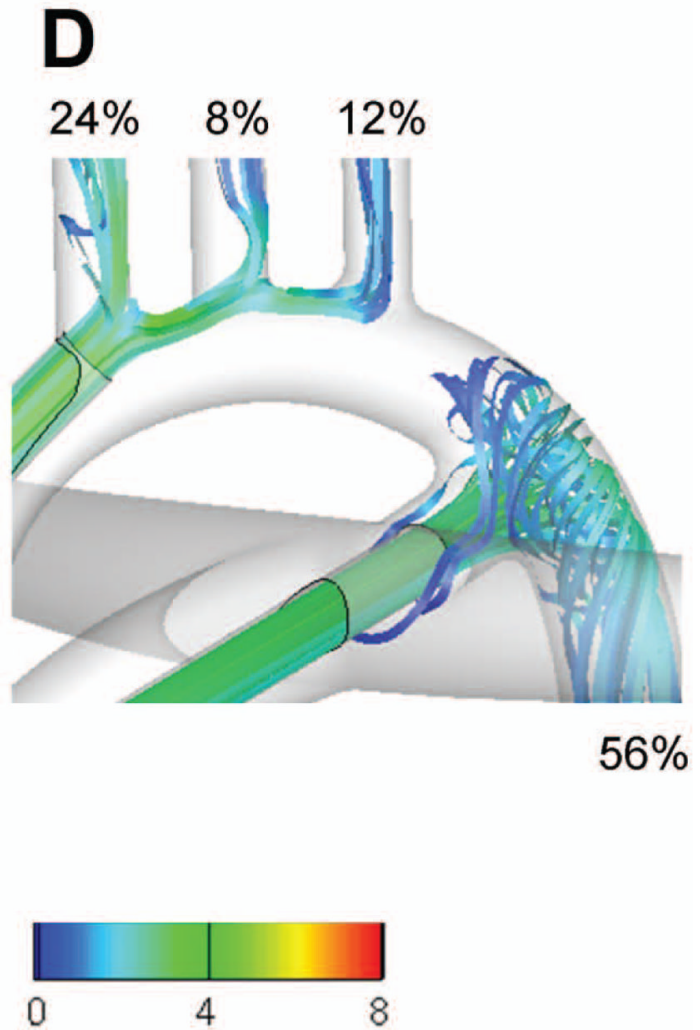

Figure 4.

Comparison of the flow distributions and velocity fields associated with the four cannulation options, under "adult" flow conditions. A: Option 1; B: Option 2; C: Option 3; D: Option 4. 


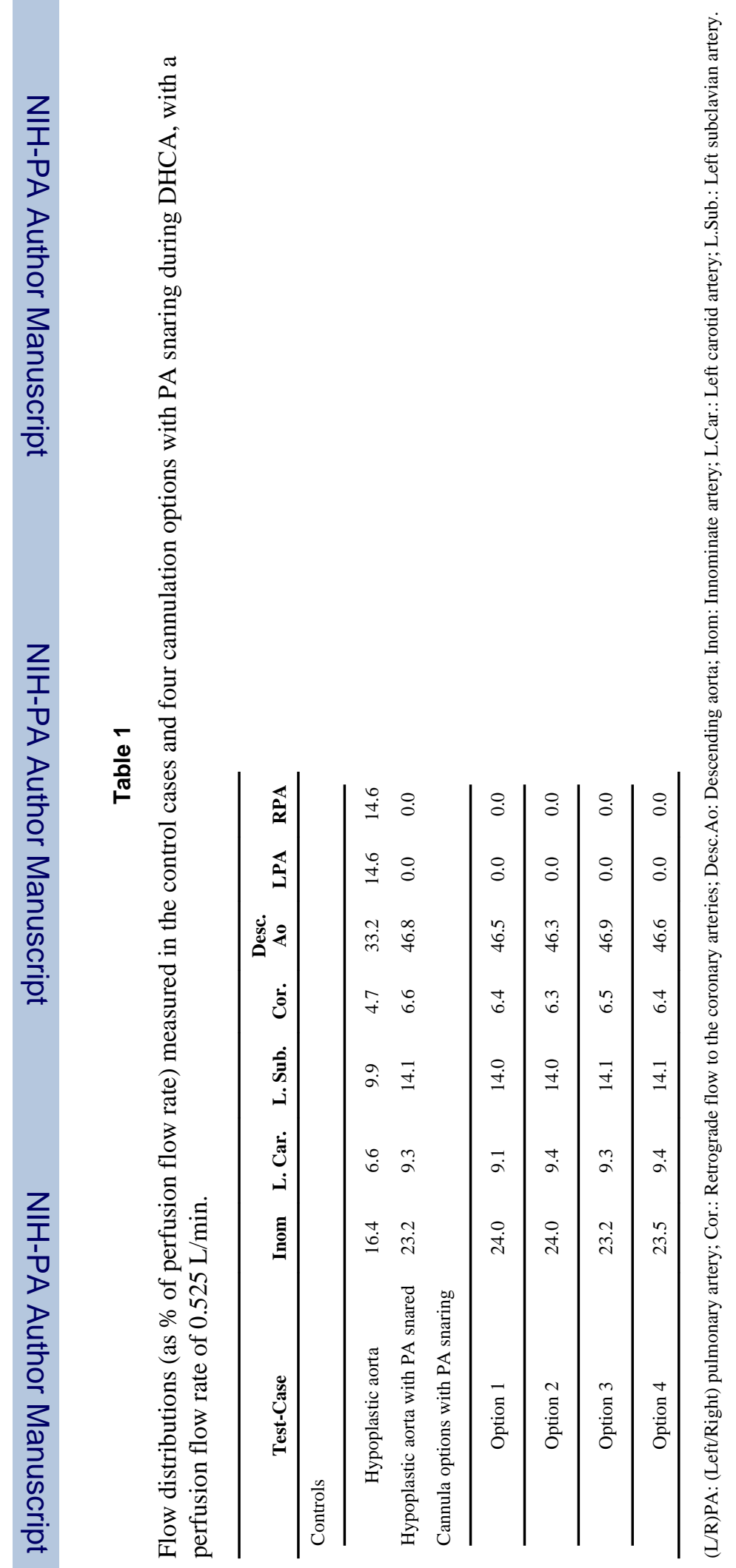

Ann Thorac Surg. Author manuscript; available in PMC 2013 August 01. 


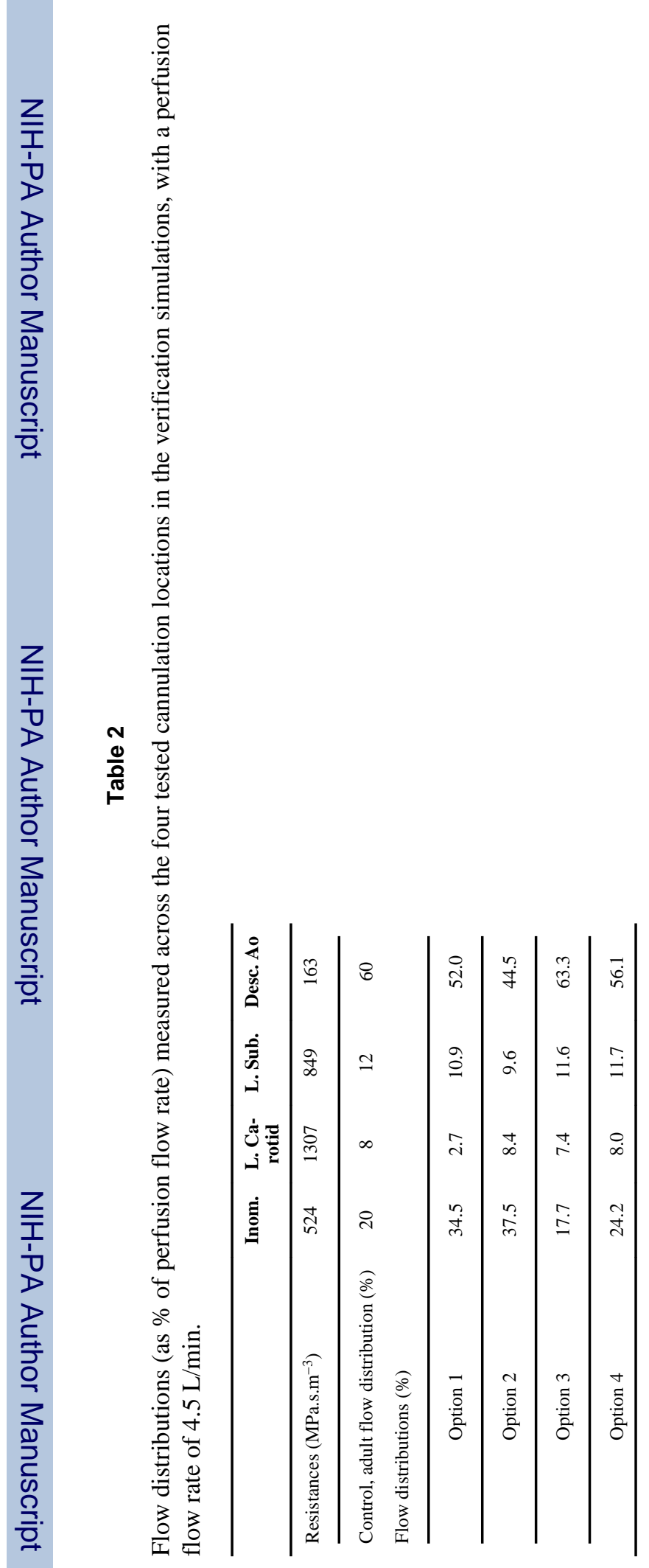

